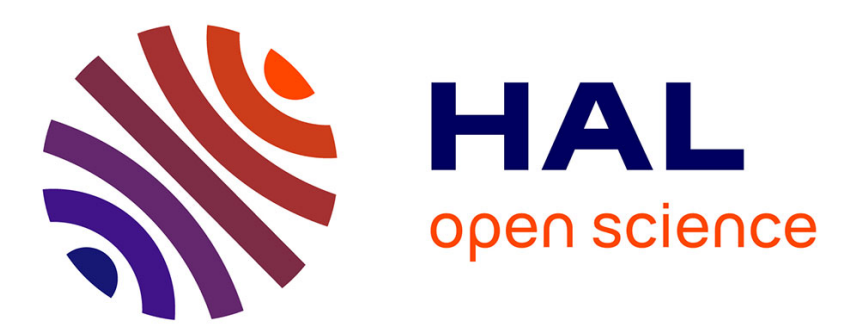

\title{
DDART, a Dynamic Dashboard for Collection, Analysis and Visualization of Activity and Reporting Traces
}

\author{
Min Ji, Christine Michel, Elise Lavoué, Sébastien George
}

\section{To cite this version:}

Min Ji, Christine Michel, Elise Lavoué, Sébastien George. DDART, a Dynamic Dashboard for Collection, Analysis and Visualization of Activity and Reporting Traces. 9th European Conference on Technology Enhanced Learning (EC-TEL 2014), Graz, Austria, 16-19 September 2014, Sep 2014, Graz, Austria. pp.440-445, 10.1007/978-3-319-11200-8_39. hal-01130922

\section{HAL Id: hal-01130922 \\ https://hal.science/hal-01130922}

Submitted on 12 Mar 2015

HAL is a multi-disciplinary open access archive for the deposit and dissemination of scientific research documents, whether they are published or not. The documents may come from teaching and research institutions in France or abroad, or from public or private research centers.
L'archive ouverte pluridisciplinaire HAL, est destinée au dépôt et à la diffusion de documents scientifiques de niveau recherche, publiés ou non, émanant des établissements d'enseignement et de recherche français ou étrangers, des laboratoires publics ou privés. 


\title{
DDART, a Dynamic Dashboard for Collection, Analysis and Visualization of Activity and Reporting Traces
}

\author{
Min $\mathrm{Ji}^{1}$, Christine Michel ${ }^{1}$, Elise Lavoué ${ }^{2}$, Sébastien George ${ }^{3}$ \\ ${ }^{1}$ Université de Lyon, CNRS \\ INSA-Lyon, LIRIS, UMR5205, F-69621, France \\ \{min.ji; christine.michel\}@liris.cnrs.fr \\ ${ }^{2}$ Magellan, IAE Lyon, Université Jean Moulin Lyon 3, France \\ elise.lavoue@univ-lyon3.fr \\ ${ }^{3}$ LUNAM Université, Université du Maine, EA 4023, LIUM, 72085 Le Mans, France \\ Sebastien.George@univ-lemans.fr
}

\begin{abstract}
Most Learning Management Systems (LMS) focus on providing rich learning materials to the learners but rarely offer possibilities to monitor and analyze their learning processes. However metacognitive strategies argue that learners can improve their learning performances by monitoring their activities, especially in the context of Project-Based Learning (PBL). Our work consists in designing tools to support metacognitive strategies. In this paper, a framework and a dynamic dashboard for PBL situations are proposed to help learners to collect, analyze and visualize the meaningful traces of their activities by themselves. The proposed framework integrates activity traces (interactions with the LMS) and reporting traces (semi-structured sentences written by the learners). The dynamic dashboard supports learners in the creation customizable indicators through a user-friendly interface.
\end{abstract}

Keywords: project-based learning, indicator, dashboard, self-regulation, metacognition.

\section{Introduction}

The World Wide Web provides opportunities for creating virtual classrooms for learners and instructors [1]. Unfortunately, most Learning Management Systems (LMS) rarely offer possibilities to monitor and analyze their learning processes to improve their learning performances. Hence, our research aims to improve learners' meta-cognition and self-regulation awareness by exploring the traces produced during the learning processes. We focus on the development of tools to help learners to monitor their own learning activities.

Our works are conducted in the context of Project-based-Learning (PBL). Michel et al. [2] found that the use of a dashboard in PBL could help the learners to develop metacognitive skills and new behaviors. Based on this fact, our general research question is how to design a dashboard to help learners to self-regulate and build metacognitive skills during their project activities.

adfa, p. 1, 2011.

(C) Springer-Verlag Berlin Heidelberg 2011 
In our previous works[3, 4], we have defined a dashboard used in the PBL context as a Project Based Learning Management System (PBLMS). We have proposed a general architecture based on the management of the traces of the project activities. This architecture combines activity traces and reporting traces into a dashboard to support the learning processes [4]. The activity traces can reflect the interactions in the PBLMS environment, while the reporting traces can record the interactions out of the PBLMS environment. These two types of traces can give a general view of the project and can support self-regulation processes specifically if the learners are able to explore them directly.

In this paper, we propose an operational dynamic dashboard for learners using both activity and reporting traces. In part 2, we introduce the existing dashboards used to support self-regulation and give a critical analysis. In part 3 we present the DDART system, a dynamic dashboard we developed. We finally conclude in part 4 and detail our future works.

\section{State of the Art}

A self-regulating dashboard should present the information about goals and activities at a glance on the shape of indicators that allow easy navigation to more complete information on analysis views [5]. So, the dashboard could be considered as a container of indicators [3]. In order to calculate the indicators, we have identified two types of traces useful to build the indicators: activity traces and reporting traces [4]. This part describes the existing simple dashboards that enable the users to use predefined indicators (all the aspects of the indicator are defined by the system developers totally) and dynamic dashboards that let the learners customize their own indicators (users can define all the aspects of indicators).

\subsection{Simple Dashboards with Predefined Indicators}

Simple dashboards are designed to help the users to monitor some specific aspects of their activities. CourseVis [1] computes graphical indicators by activity traces in WebCT. TrAVis [6] is a reflective tool that also uses activity traces to give students information about the way they carry out discussions or other collaborative activities. Students have access to the tracking data repository. Study desk [7] supports the monitoring of the learners' learning processes by evaluating the progresses and tasks from activity traces. Feeler [8] uses personal physical data to reflect the relationship between learners' learning performances and well beings with the aim of fostering reflection and awareness. NAVI Badgeboard [9] aims at improving individual awareness and reflection of personal activities through visualizing learners' communication activities by "badge" presentation.

The system gStudy [10] is a collaborative learning platform. The traces collected are the log files and the messages in the module gChat. All the traces are analyzed by the module LogAnalyser. Radar [11] is a peer feedback tool that provides users with anonymous information on how their cognitive and social behavior is perceived by 
themselves, their peers and the group as a whole. Reflector [11] is a reflection tool that computes learner's answers about five reflective questions. PCO-vision [2] is a dashboard that supports self-regulation by offering a global view on objectivesactions-results. The learners self-declare their goals and the way they carry out activities. They can explore these reporting traces to self-regulate themselves.

\subsection{Dynamic Dashboards with Customizable Indicators}

Dynamic dashboards offer three customization levels for the indicators. In a low level, users can set some simple parameters of the indicators; in a medium level, users can define the calculation functions and change the visualizations of the indicators; in a high level, learners can manipulate the traces used in the indicators. Navi Surface [9] has a low level of customization. It uses the same principle of badges presented with NAVI Badgeboard (mentioned in last section). Users can specify the names of the group members in order to view how the group badges acquisitions arise. The only visualization mode is badges. The Academic Analytics Tool (AAT) [12] supplies tutors with medium customization functions by allowing them to extract specific information from the activity traces and to select the calculation methods through a SQL query GUI. Specific computer skills are needed (write SQL) and the only visualization mode of the results is tables. GINDIC [13] supports the generation of high customizable indicators by using activity traces but requires the users to have a computer background because many parameters need to be set. The TBS-IM system [14] uses the concept of modeled trace (M-Trace) to enable tutors to select, transform and visualize activity traces produced by Moodle. The customization process is high. It offers various visualization modes to users but tutors are required to have some computer skills to understand how to manipulate the traces and to define parameters.

\subsection{Summary / Critical Analysis of Existing Dashboard}

The previously presented dashboards can be compared from four aspects: target user, trace type, customization level and computer skills requirements.

- Most dynamic dashboards are dedicated to tutors and researchers while most simple dashboards are supplied to learners. Learners have no means to self-regulate their activities and to build metacognitive skills.

- Most dashboards explore the activity traces to analyze the learning processes while the reporting traces are ignored. The whole learning processes can be observed completely only by combining the two types of traces.

- Most dashboards don’t supply the customization functions or just supply some low customization functions.

- The higher the customization level is, the higher the computer background requirements are. There is no simple but high-dynamic dashboard for learners.

Based on the above analysis, we propose in the next section a dynamic dashboard based on the LMS Moodle. 


\section{DDART System}

DDART is a Dynamic Dashboard Based on Activity and Reporting Traces. This system allows learners to create customizable indicators related to their activities. We choose to implement it as a plug-in of the Moodle platform. It is based on a four steps framework detailed in each part of this section: data collection, data integration, data calculation and data visualization.

\subsection{Data Collection}

Two types of traces are collected: activity traces and reporting traces. We have classified the activities in PBL into three categories: internal activities, external activities and non-instrumented activities [4]. We extract parts of the internal activities data, recorded directly by Moodle, to constitute the activity traces. These traces come from the use of five modules integrated in the platform (wiki, chat room, forum, private message and resource) and of two modules we developed: a reporting tool and DDART. The reporting traces are recorded when learners write reports in the reporting tool [4] to declare their non-instrumented activities and external activities. The reports are composed of semi-structured sentences.

\subsection{Data Integration}

This step aggregates the two types of traces together and generates a primary trace (PT). A primary trace is defined as "a trace model and a set of untransformed traces instances according to this model" [14]. It is the source data for creating the indicators. Based on our PBL context, we propose a task-oriented pattern model to store the primary traces:

$$
\mathrm{PT}=\{\mathrm{Ta}, \mathrm{L}, \mathrm{C}, \mathrm{V}, \mathrm{To}, \mathrm{BT}, \mathrm{ET}, \mathrm{P}\}
$$

With:

- Ta: the task carried out by learners during the project; L: the learner who carries out the task; C: the category of the task; V: the value (detailed content) of the task; To: the tools supplied in Moodle to help learners to complete the project; BT and ET: begin time and end time of the task; P: the place at where the task was done.

A filter function is supplied to help learners to set the constraints of learners, time, places, tools and tasks so as to have a smaller set of traces.

\subsection{Data Calculation}

The Fig. 1 presents the interface of the indicator design process. This interface is composed of three parts: (1) the "parameters" part ( see Fig. 1.a ) contains the list of all the parameters which are available for creating an indicator, (2) the "calculation" part ( see Fig. 1.b and c ) allows learners to specify the parameters and presents the 
view of the indicator results and (3) the "visualization modes" part ( see Fig. 1.d ) supplies ten presentation models to learners to choose.

This user-friendly interface is WYSIWYG (what you see is what you get) and the results can be calculated on the fly, without delay, so that learners can adjust the parameters all the time. It allows learners to create the indicators by dragging and dropping the parameters and the visualization mode. Learners can manipulate five entities $\mathrm{E}=\{\mathrm{L}$, To, Ta, Time, P $\}$ from the PT model. They can cross the entities together, select the type of value to consider (frequency, time interval, content and description) or do some mathematical calculations (sum, means, etc.). The cross operator calculates the cartesian product E1×E2 as follow:
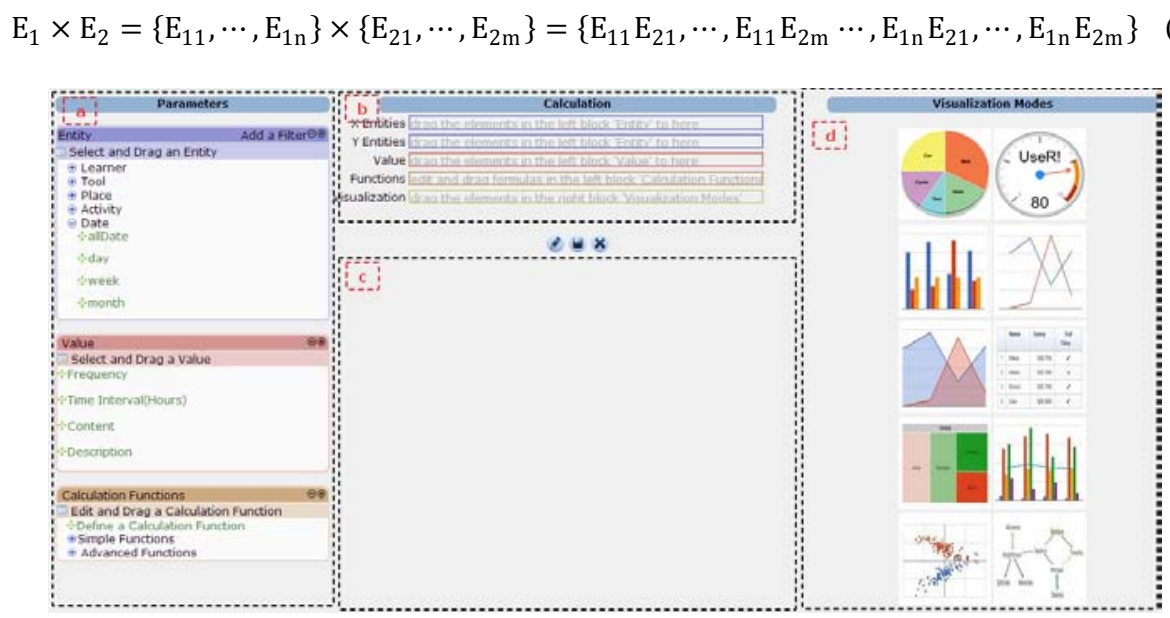

Fig. 1. The main page for trace transformation and indicator calculation

\subsection{Data Visualization}

DDART offers ten visualization modes for learners: pie chart, bar chart, line chart, gauge chart, social network, scatter chart, area chart, table, tree map and combo chart. We believe that different visualization modes can help learners to obtain different information from different dimensions. We imported Google Chart API to generate these visualizations automatically. All the created indicators are presented in the learners' own dashboards. Learners can follow their project and learning by observing their own customizable dashboards.

\section{Conclusion}

In this paper, we have presented the DDART system, a dynamic dashboard integrated to Moodle. This system can help learners to collect, analyze and visualize their traces in the form of meaningful indicators. By providing learners with DDART, we encourage them to explore information by themselves and learn how to regulate their learning activities. Considering our future research work, we will conduct an experiment in 
a real PBL situation to test the usability and the utility of DDART as a support for learners' self-regulation.

\section{References}

1. Mazza, R., Dimitrova, V.: CourseVis: A graphical student monitoring tool for supporting instructors in web-based distance courses. International Journal of Human-Computer Studies. 65(2), p.125-139 (2007).

2. Michel, C., Lavoué, E., Pietrac, L.: A Dashboard to Regulate Project-Based Learning. 21st Century Learning for 21st Century Skills. Berlin, Heidelberg: Springer Berlin Heidelberg. p. 250-263 (2012).

3. Ji,M.: Ergonomic Study of Existing Project-Based Learning Management System. The Fourth Young Researchers Conference on Information Environment for Human Learning. p. 57-62 (2012).

4. Ji, M., Michel, C., Lavoué, E., and George, S.: An Architecture to Combine Activity Traces and Reporting Traces to Support Self-Regulation Processes. IEEE 13th International Conference on Advanced Learning Technologies (ICALT). p. 87-91. IEEE (2013).

5. Michel, C., Lavoué, E.: KM and Web 2.0 Methods for Project-Based Learning. MEShaT: a Monitoring and Experience Sharing Tool. Multiple Perspectives on Problem Solving and Learning in the Digital Age. p. 49-66. Ifenthaler D., Isaias P., Spector J.M., Kinshuk, Sampson D., Heidelberg (2011).

6. May, M., George, S., Prévôt, P.: TrAVis to Enhance Students' Self-monitoring in Online Learning Supported by Computer-Mediated Communication Tools. Computer Information Systems and Industrial Management Applications, vol. 3, p. 623-634 (2011).

7. Narciss, S., Proske, A., Koerndle, H.: Promoting self-regulated learning in web-based learning environments. Computers in Human Behavior, 23(3), p. 1126-1144 (2007).

8. Durall, E., Toikkanen, T.: Feeler: feel good and learn better. Third International Workshop on Awareness and Reflection in Technology-Enhanced Learning (ARTEL 2013). p.83 89 (2013).

9. Charleer, S., Klerkx, J., Santos, J. L., Duval, E.: Improving awareness and reflection through collaborative, interactive visualizations of badges. Third International Workshop on Awareness and Reflection in Technology-Enhanced Learning (ARTEL 2013), p.83 89 (2013).

10. Winne, P. H., Hadwin, A. F., Gress, C.: The learning kit project: Software tools for supporting and researching regulation of collaborative learning. Computers in Human Behavior, 26(5), p. 787-793 (2010).

11. Phielix, C., Prins, F. J., Kirschner, P. A.: Group Awareness of Social and Cognitive behavior in a CSCL Environment. 9th International Conference of the Learning Sciences-. International Society of the Learning Sciences. Vol.1, p. 230-237 (2010).

12. Graf, S., Ives, C., Rahman, N., Ferri, A.: AAT: a tool for accessing and analysing students' behaviour data in learning systems. The 1st International Conference on Learning Analytics and Knowledge, p. 174-179 (2011).

13. Gendron, É.: Cadre conceptuel pour l'élaboration d'indicateurs de collaboration àpartir des traces d'activité. University of Claude Bernard Lyon I (2010).

14. Djouad, T., Benmohammed, M., Mille, A.: Indicators computation from modeled traces in the context of computer Human Learning environment. In International Symposium on Modelling and Implementation of Complex Systems, Constantine, Algérie, p. 1-7 (2010). 